\title{
De-Embedding-Ansatz zur Messung nichtidealer Kapazitäten mit kommerziellen Kapazitätssensoren
}

\author{
De-embedding-approach to measure non-ideal capacitances with commercially available \\ capacitance-sensors
}

DOI $10.1515 /$ teme-2020-0016

Zusammenfassung: Verlustbehaftete Kapazitäten sind für kommerzielle Kapazitätssensoren, die nur einen Kapazitätswert ausgeben, problematisch: der parasitäre ohmsche Widerstand parallel zur Kapazität verfälscht das Messergebnis. Bei Messungen an Flüssigkeiten im Rahmen der elektrischen Kapazitätstomographie (ECT) kann der Einfluss gravierend werden. Mit erhöhtem Messaufwand könnte man die ECT zur elektrischen Impedanztomographie (EIT) erweitern und damit sowohl die Kapazität als auch den Widerstand der verlustbehafteten Kapazität messen. Als aufwandsarme Alternative stellen wir hier einen modellbasierten De-Embedding-Ansatz vor. Er ertüchtigt kommerzielle Kapazitätssensoren, die die Kapazität über die Messung einer Entladezeitkonstante bestimmen, auch mit stärker verlustbehafteten Kapazitäten zurechtzukommen. Der Ansatz beruht auf dem Ersatzschaltbild der realen Messsituation, dessen zugehörige Differentialgleichung im Zeitbereich gelöst wird. Durch Variation der gesuchten Kapazität und des gesuchten Widerstands wird die vom Sensor ermittelte Zeitkonstante über die Anzahl der nacheinander durchgeführten Lade-/Entladevorgänge (Perioden) ermittelt. Das Verfahren wird an einem diskreten Aufbau aus konzentrierten Elementen und an einer typischen ECT-Messsituation getestet. Die ermittelten Zeitkonstanten bei einer realen ECT-Messsituation mit Leitungswasser als zu vermessendem Medium zeigen einen Unterschied zum diskreten Aufbau mit den konzentrierten Elementen.

\footnotetext{
*Korrespondenzautor: Christoph Kandlbinder-Paret, Lehrstuhl für Mess- und Regeltechnik, Universität Bayreuth, Universitätsstraße 30, 95447 Bayreuth, E-Mail: mrt@uni-bayreuth.de
} Johannes Häring, Alice Fischerauer, Gerhard Fischerauer, Lehrstuhl für Mess- und Regeltechnik, Universität Bayreuth, Universitätsstraße 30, 95447 Bayreuth
Schlüsselwörter: Kapazitätssensoren, ECT, DeEmbedding.

Abstract: Lossy capacitances are problematic for commercial capacitance sensors which only output a capacitance value: a parasitic resistance parallel to the capacitance influences the measurement result. The influence can be serious when measuring liquids using electrical capacitance tomography (ECT). With increased measurement effort, ECT could be expanded to electrical impedance tomography (EIT) and thus both the capacitance and the resistance of the lossy capacitance could be measured. As a low-cost alternative, we present a model-based deembedding approach. It enhances commercial capacitance sensors, which determine the capacitance by measuring the discharge time, to cope with lossy capacitances. The approach is based on the equivalent circuit of the real measurement situation, the associated differential equation of which is solved in the time domain. The discharge time determined by the sensor is determined by varying the capacitance and the resistance sought by the number of charging/discharging processes (periods) carried out in succession. The method is tested on a discrete structure consisting of concentrated elements and on a typical ECT measurement situation. The discharge time determined for the real ECT measurement situation with air and water as the media to be measured were all within the uncertainty range of the respective measurement series. Therefore, there was no discernible difference between the different media in the discharge time, which is justified by the small change in capacitance.

Keywords: Capacitance sensors, ECT, de-embedding.

\section{Einleitung}

Kommerziell erhältliche Sensoren zur Messung der elektrischen Kapazität geben lediglich eine Kapazität als Mess- 
wert aus. In Anwesenheit von Verlusten wirkt aber eine reale Kapazität wie die Parallelschaltung einer idealen Kapazität mit einem ohmschen Widerstand. Der Verlustwiderstand verursacht damit eine Messabweichung bei der Kapazitätsmessung. Diese Abweichung wird beispielsweise im Datenblatt des kommerziellen ICs AD7746 in Abhängigkeit von der Größe des Widerstands angegeben. Für ein Multielektrodensystem wurde bereits gezeigt, dass dadurch Rückschlüsse auf die Leitfähigkeit des Mediums zwischen den Elektroden gezogen werden können [3]. Eine solche Signalverarbeitung ist von hohem Interesse in der elektrischen Kapazitätstomografie (ECT) [6].

\section{Methodik}

Zur Herleitung eines De-Embedding-Verfahrens, das den Einfluss des Verlustwiderstands einer realen Kapazität beseitigt, gehen wir von dem Kapazitätssensor PCap02 von ams aus. Wenn man den Messaufwand nicht erhöhen (und beispielsweise Impedanzen statt Kapazitäten messen) will, bleibt einem nur der Rückgriff auf eine modellbasierte Signalverarbeitung. Man stelle sich vor, dass die Permittivitätsverteilung im Inneren eines Behälters oder eines Rohres mit der ECT ermittelt werden soll [1, 2]. Ist das zu untersuchende Medium kein ideales Dielektrikum, sondern besitzt auch eine elektrische Leitfähigkeit, „sieht“ der Kapazitätssensor eine verlustbehaftete (effektive) Kapazität. Abb. 1a) stellt das elektrische Ersatzschaltbild (ESB) einer solchen Situation dar. Darin beschreiben die Spannung $U_{0}$ und die Widerstände $R_{0}$ und $R_{D}$ die Klemmeneigenschaften des PCap02 (Helmholtz-Ersatzspannungsquelle). Der rechte Netzwerkteil wird über $R_{0}$ aufgeladen und über $R_{D}$ entladen. Die Kapazität $C_{X}$ ist die eigentlich interessierende Messgröße, aus der man Informationen über die Permittivität eines beobachteten Mediums ( $m e$ dium under test, MUT) gewinnt. Der Parallelwiderstand $R_{X}$ entsteht wegen der endlichen MUT-Leitfähigkeit. $C_{S}$ modelliert die auftretenden Streukapazitäten gegen Masse, und $C_{W}$ repräsentiert die Wandkapazität infolge der Isolationsschicht zwischen den Messelektroden und dem MUT. Bei idealem Dielektrikum $\left(R_{X}=0\right)$ würden $C_{S}$, $C_{W}$ und $C_{X}$ eine ideale Kapazität bilden (noch besser wäre natürlich zusätzlich $C_{S}=C_{W}=0$, dann würde $C_{X}$ ungestört gemessen).

Der PCap02 arbeitet nach dem Lade/Entladeverfahren. Dabei wird der in Abb. 1a) gezeigte Schalter periodisch mit der Frequenz $f=143 \mathrm{kHz}$ umgelegt, was bei $R_{X}=0 \mathrm{zu}$ dem in Abb. 1b) dargestellten Spannungsverlauf $U_{S}(t)$ über der Kapazität $C_{S}$ führt a)

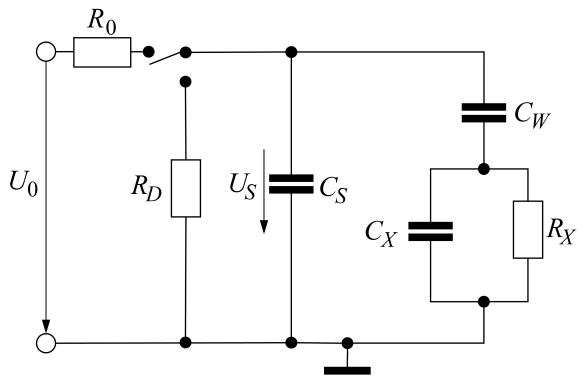

b)

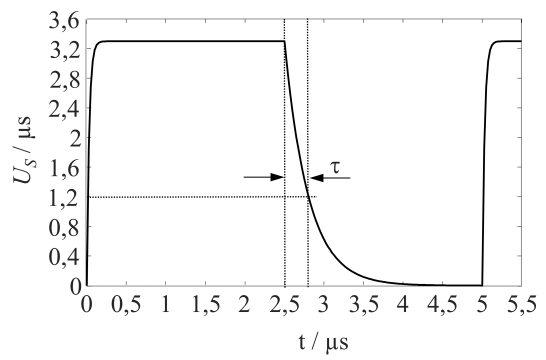

Abb. 1: Messsituation bei der ECT: a) Ersatzschaltbild mit dem PCap02 bei nichtidealem Dielektrikum. Siehe Text zur Erläuterung. b) Spannungsverlauf im PCap02 bei idealem Dielektrikum $\left(R_{X}=0, R_{D}=10 R_{0}\right)$.

(für eine ideale Kapazität wird $R_{0} \ll R_{D}$ gewählt). Es wird die Zeitkonstante $\tau_{\text {mess }}$ gemessen, also die Zeit, nach der $U_{S}(t)$ in der Entladephase von $U_{0}=3,3 \mathrm{~V}$ bis auf einen festgelegten Schwellwert gefallen ist, und mit der Zeitkonstante $\tau_{\text {ref }}$ einer bekannten Referenzkapazität $C_{\text {ref }}$ verglichen[4]. Da die Messkapazität und die Referenzkapazität über den gleichen Widerstand $R_{D}$ entladen werden, gilt $\tau_{\text {mess }} / \tau_{\text {ref }}=C_{\text {mess }} / C_{\text {ref }}$. Damit ist die Kapazitätsmessung auf zwei Zeitmessungen zurückgeführt.

Bei $R_{X} \neq 0$, also bei Gültigkeit des vollständigen ESB von Abb. 1a), folgt der Lade-/Entladevorgang nicht mehr einer Differentialgleichung (DGL) erster Ordnung, wie sie Abb. 1b) zugrunde liegt, sondern einer DGL zweiter Ordnung. Die Lösung dieser DGL für eine Sprunganregung der zuvor ungeladenen Kapazität $C_{S}$ bei $t=0$ lautet:

$$
\begin{aligned}
u_{S}(t)= & \left(K_{1}-K_{2} T_{1}\right) \mathrm{e}^{-t / T_{1}}-\left(K_{1}-K_{2} T_{2}\right) \mathrm{e}^{-t / T_{2}} \\
& +K_{2}\left(T_{1}-T_{2}\right)
\end{aligned}
$$

mit

$$
\begin{aligned}
K_{1} & =\frac{T_{1} T_{2}}{T_{1}-T_{2}} \dot{u}_{X}\left(0^{+}\right)\left(1+\frac{C_{X}}{C_{W}}\right), \\
K_{2} & =\frac{K_{1}}{\left(C_{W}+C_{X}\right) R_{X}} .
\end{aligned}
$$

Darin ist $\dot{u}_{X}\left(0^{+}\right)$die zeitliche Ableitung der Spannung an der gesuchten Kapazität $C_{X}$ unmittelbar nach der Sprunganregung:

$$
\dot{u}_{X}\left(0^{+}\right)=\frac{U_{0} C_{W}}{R_{0}\left(C_{S}\left(C_{X}+C_{W}\right)+C_{X} C_{W}\right)},
$$




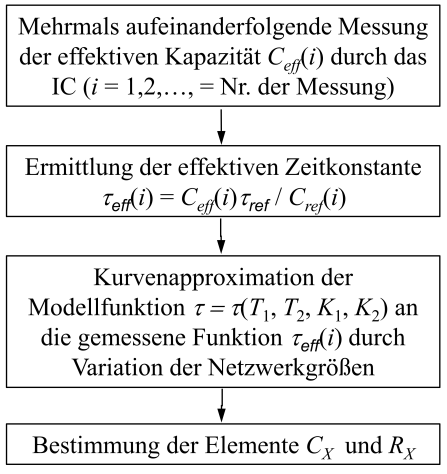

Abb. 2: Ablauf des verwendeten De-Embedding-Ansatzes.

und die Zeitkonstanten $T_{1}, T_{2}$ sind festgelegt durch:

$$
\begin{aligned}
\frac{1}{T_{1,2}}= & \frac{R_{X}\left(C_{X}+C_{W}\right)+R_{0}\left(C_{W}+C_{S}\right)}{2 R_{X} R_{0}\left(C_{X}\left(C_{W}+C_{S}\right)+C_{W} C_{S}\right)} \\
& \mp \frac{\sqrt{T_{3}^{2}+T_{4}^{2}}}{2 R_{X} R_{0}\left(C_{X}\left(C_{W}+C_{S}\right)+C_{W} C_{S}\right)}
\end{aligned}
$$

mit

$$
\begin{aligned}
& T_{3}=\left(R_{X} C_{X}-R_{0}\left(C_{W}+C_{S}\right)\right) \text { und } \\
& T_{4}=\sqrt{R_{X} C_{W}\left(R_{X}\left(2 C_{X}+C_{W}\right)+2 R_{0}\left(C_{W}-C_{S}\right)\right)} .
\end{aligned}
$$

Es ist offensichtlich, dass die aus dem Spannungsverlauf vom PCap02 gemessene Entladezeit $\tau_{\text {eff }}$ mehr Details enthält als die Lade-/Entladekurve eines einfachen RC-Glieds. Der Ansatz des De-Embeddings ist in Abb. 2 beschrieben. Der IC gibt nicht die Entladezeitkonstante $\tau_{\text {eff }}$ aus, sondern die daraus berechnete Kapazität $C_{\text {eff }}$. Durch die mehrmals aufeinanderfolgende Messung der effektiven Kapazität $C_{\text {eff }}(i)$ durch das IC mit $i=1,2, \ldots$ entsprechend der Nummer der Messung kann wieder auf die effektive Zeitkonstante $\tau_{\text {eff }}(i)=C_{\text {eff }}(i) \cdot \tau_{\text {ref }} / C_{\text {ref }}$ zurückgerechnet werden. Mittels einer Kurvenapproximation der Modellfunktion $\tau=\tau\left(T_{1}, T_{2}, K_{1}, K_{2}\right)$ an jedes der $\tau_{\text {eff können }}$ daraufhin die Elemente $C_{X}$ und $R_{X}$ näherungsweise bestimmt werden.

Abb. 3a) zeigt den Verlauf der Spannung $U_{S}(t)$ für die zehn ersten Perioden nach Start einer Messung an dem in Abb. 1a) dargestellten Messobjekt (nichtidealer Kondensator). Der PCap02 geht weiterhin vom Zeitverhalten eines einfachen RC-Gliedes aus und interpretiert daher die Lade-/Entladekurven falsch. Die von dem IC bestimmte effektive Zeitkonstante $\tau_{\text {eff }}(i)$ hängt dadurch von der Schaltperiode und natürlich von $C_{X}$ und $R_{X}$ ab (Abb. 2b). Aus diesem Verhalten der scheinbaren Zeitkonstanten lassen sich Aussagen über $C_{X}$ und $R_{X}$ ableiten, sodass beide Größen bestimmbar werden. Daher ist der Verlauf der Zeitkonstante bei den folgenden Betrachtungen entscheidend. a)

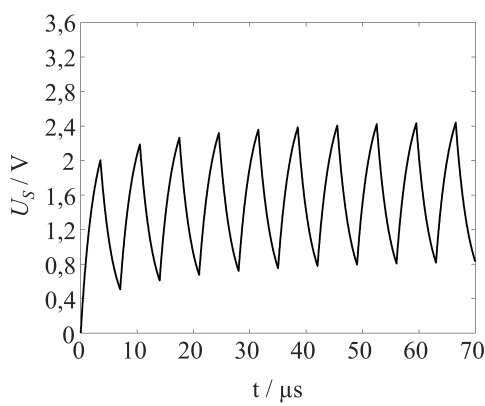

b)

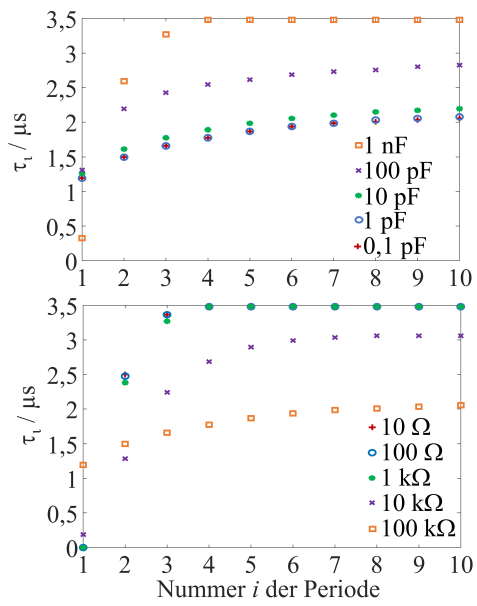

Abb. 3: Modellierte Kapazitätsmessung mit dem PCap02 bei nichtidealer Kapazität. a) Spannungsverlauf $U_{S}(t)$ über zehn Schaltperioden für das ESB von Abb. 1a) für $U_{0}=3,3 \mathrm{~V}$ und $f=143 \mathrm{kHz}$. b) Vom PCap02 ausgegebene scheinbare Zeitkonstante in Abhängigkeit von der Schaltperiode seit Beginn der Messung bei Variation von $C_{X}$ (oben; dabei war $R_{X}=100 \mathrm{k} \Omega$ ) bzw. $R_{X}$ (unten; dabei war $C_{X}=0,1 \mathrm{pF}$ ). Werte der weiteren ESBElemente: $R_{D}=R_{0}=30 \mathrm{k} \Omega ; C_{D}=100 \mathrm{pF} ; C_{W}=160 \mathrm{pF}$.

\section{Messung}

Zur Verifizierung der Methode wurde sie auf ein diskret mit konzentrierten Elementen realisiertes Messobjekt wie in Abb. 1a) angewandt. Der PCap02 wurde mit $R_{D}=R_{0}=30 \mathrm{k} \Omega$ und einem Funktionsgenerator (HAMEG HM 80306) nachgebildet, um vollständige Kontrolle über diesen Teil des Messaufbaus zu behalten. Die über dem Kondensator $C_{S}$ bei Vorgabe einer Rechteckschwingung abfallende Spannung wurde mit einem Oszilloskop (LeCroy WaveAce 202) gemessen. Die Zeitkonstante wurde so wie in Abb. 3b) ermittelt. Zur Feststellung der Wiederholgenauigkeit der Messung wurden zehn Wiederholungsmessungen aufgenommen und der Mittelwert zusammen mit der Unsicherheit auf einem Konfidenzniveau von $95 \%$ bestimmt [5, S. 31-47]. Zusätzlich wurde der theoretisch erwartete Zeitverlauf berechnet. 
Die Messergebnisse stimmen sehr gut mit den theoretischen Erwartungen überein (Abb. 4). Dazu musste allerdings ein Offset von 0,2 V für die gemessene Kurve eingeführt werden, um einen Spannungs-Offset des Funktionsgenerators auszugleichen. Die Kapazität $C_{S}$ war im Aufbau diskret mit $100 \mathrm{pF}$ realisiert, der optimale Wert für die Anpassung von Messung und Rechnung betrug allerdings $150 \mathrm{pF}$; der Unterschied rührt von parasitären Leitungskapazitäten her.

Die aus den Lade-/Entladekurven mit der PCap02Fiktion einer idealen Kapazität abgeleitete Zeitkonstante stimmt zwischen Messung und Rechnung erwartungsgemäß recht gut überein (Abb. 3b)). Bei längerer Messung (höherer Periodenindex) macht sich der Einfluss des Messrauschens zunehmend bemerkbar. Man erkennt ihn auch an der mit der Zeit anwachsenden Breite des Konfidenzintervalls in Abb. 3a).

Insgesamt bestätigt der überzeugende RechnungsMessungs-Vergleich, dass die experimentell beobachteten Eigenschaften der nichtidealen Kapazität bei einer ECTMessung mit nur geringen Abweichungen durch das Netzwerkmodell von Abb. 1a) nachgebildet werden können.

Für die Verifizierung bei einer typischen ECTMesssituation mit verteilten Kapazitäten wird ein Elektrodenring mit 16 Elektroden ausgewählt, von denen zwei gegenüberliegende Elektroden kontaktiert werden. Der Innendurchmesser des konkret realisierten Rings betrug $80 \mathrm{~mm}$. Die Elektroden des Aufbaus waren gegen das Medium mit einer $1 \mathrm{~mm}$ dicken Schicht aus Polycarbonat isoliert (dies wird in Abb. 1a) durch die Kapazität $C_{W}$ beschrieben). Der Aufbau war von einer äußeren, auf Masse befindlichen Schirmung umgeben. Alle anderen Elektroden außer den zwei Elektroden, die die Anschlüsse der zu messenden Kapazität bildeten, waren potentialfrei. Ein Bild des Aufbaus ist in Abb. 5a) zu sehen. Als MUT im Inneren diente Leitungswasser.

Der resultierende Verlauf der scheinbaren Zeitkonstanten analog zu Abb. 4b) ist in Abb. 5b) dargestellt. Es ist erkennbar, dass sich die Zeitkonstante mit der Periodennummer ändert und zu einem Endwert hin konvergiert. Im Vergleich zu Abb. 4b) lässt sich auch ein Unterschied im erreichten Endwert und in der Steigung über die jeweiligen Perioden erkennen.

\section{Ergebnisse und Diskussion}

Insgesamt lässt sich durch das beschriebene Verfahren mit einem kostengünstigen Kapazitätssensor, bei dem lediglich die Firmware und keine Hardware geändert werden
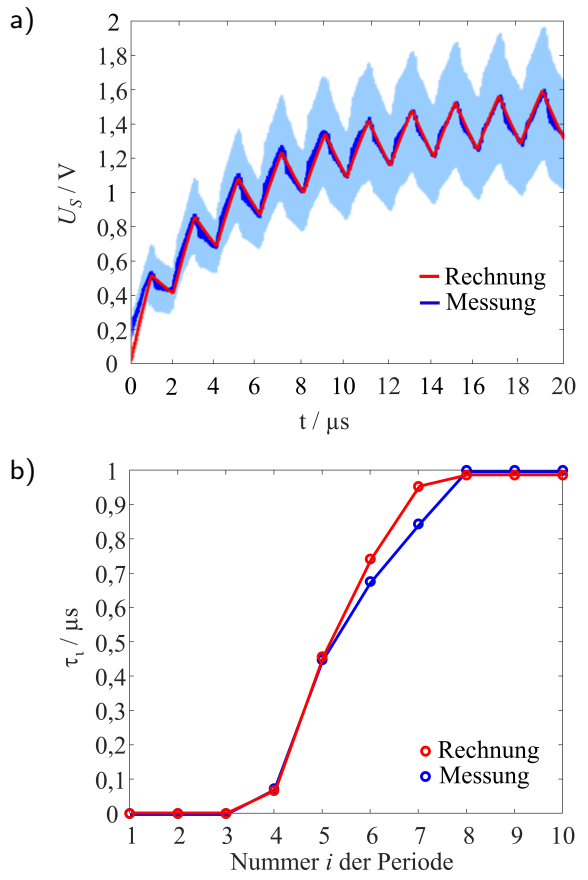

Abb. 4: Berechnete und mit dem Oszilloskop aufgenommene Spannungskurve $U_{S}(t)$ sowie ihre Auswertung bei der Messung einer verlustbehafteten Kapazität (ESB von Abb. 1a) nach dem Verfahren des PCap02. a) Berechneter Verlauf (rote Kurve) und Mittelwert des gemessenen Verlaufs (blaue Kurve, mit $95 \%$-Konfidenzintervall) der Spannung $U_{S}(t)$ über zehn Schaltperioden. b) Aus a) ermittelte scheinbare Zeitkonstante in Abhängigkeit von der Schaltperiode seit Beginn der Messung für den berechneten und den gemessenen Zeitverlauf (rote bzw. blaue Kreise). Die durchgezogenen Linien dienen lediglich der Führung des Auges. Zahlenwerte aller Parameter: $R_{X}=100 \mathrm{k} \Omega$; $C_{X}=10 \mathrm{pF} ; R_{D}=R_{0}=30 \mathrm{k} \Omega ; C_{D}=150 \mathrm{pF} ; C_{W}=100 \mathrm{pF}$; $U_{0}=3,3 \mathrm{~V}$ und $f=500 \mathrm{kHz}$.

müssen, auf eine Änderung der eigentlich interessierenden Größe $C_{X}$ schließen, obwohl sie durch die Einbettung in ein umgebendes Netzwerk (aus $R_{X}, C_{W}$ und $C_{S}$ ) nicht direkt beobachtbar ist. Das De-Embedding erlaubt es zudem, auch auf die Höhe der Änderung des Verlustterms $R_{X}$ zu schließen. Auf dieser Grundlage werden ECT-Systeme, die zur Messung vieler Kapazitäten mehrere Kapazitätssensoren umfassen, kostengünstiger. Sie werden außerdem in begrenztem Maße befähigt, neben Permittivitäts- auch Leitfähigkeitsverteilungen in einem Raumgebiet zu bestimmen. Aus Zeit- und Platzgründen werden hier die Ermittlung der Kapazität und des Widerstands und weitere Messungen nicht weiter aufgeführt. 
a)

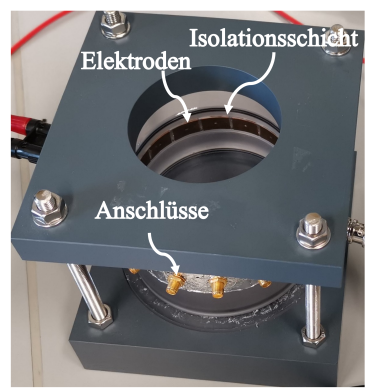

b)

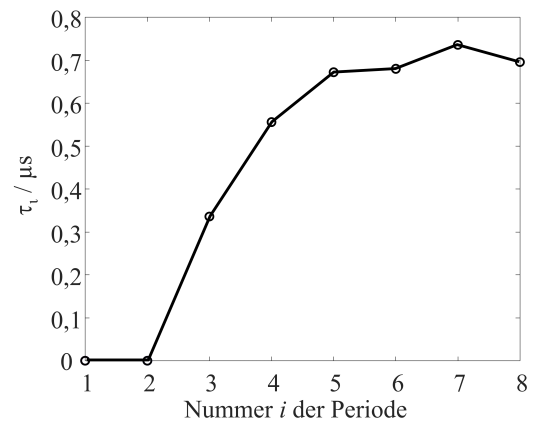

Abb. 5: Typische ECT-Messsituation. a) Aufbau des verwendeten Elektrodenrings (Details siehe Text). b) Aus dem Zeitverlauf ermittelte scheinbare Zeitkonstante in Abhängigkeit von der Schaltperiode seit Beginn der Messung für den verwendeten Aufbau bei $f=500 \mathrm{kHz}$ und $R_{D}=R_{0}=30 \mathrm{k} \Omega$. Als MUT wurde Leitungswasser verwendet. Die durchgezogene Linie dient lediglich der Führung des Auges.

\section{Literatur}

[1] C. Gunes, Q. Marashdesh und F. Teixeira. A comparison between electrical capacitance tomography and displacementcurrent phase tomography. IEEE Sens. J., 17(24):8037-8046, 2017.

[2] Y. D. Jiang und M. Soleimani. Capacitively coupled impedance imaging based on wideband phase measurement. In Proc. of 9th World Congr. Industr. Proc. Tomography (WCIPT), S. 3-9, 2018.

[3] C. Kandlbinder-Paret, A. Fischerauer, A. Horn und G. Fischerauer. Berührungslose Permittivitäts- und Leitfähigkeitsmessung mit einem Mehrelektrodensystem. In Tagungsband 19. GMA/ITG-Fachtagung Sensoren und Messsysteme 2018, S. 165-169, 2018.

[4] N. N. PCap02A - Single-chip Solution for Capacitance Measurement Volume 1: General Data and Front-end Description (Vers. 1.6). ams Group, Mai 2014.

[5] E. Schrüfer, L. Reindl und B. Zagar. Elektrische Messtechnik. Carl Hanser Verlag München, 11. Auflage, 2014.

[6] M. Zhang und M. Soleimani. Simultaneous reconstruction of permittivity and conductivity using multi-frequency admittance measurement in electrical capacitance tomography. Meas. Sci. Technol., 27(2), 2016. 10.1088/0957-0233/27/2/025405. 